\title{
The combination of positive and negative feedback loops confers exquisite flexibility to biochemical switches
}

\author{
Benjamin Pfeuty and Kunihiko Kaneko \\ Department of Pure and Applied Sciences, University of Tokyo, Tokyo 153-8902, Japan \\ and \\ ERATO Complex Systems Biology project, JST, Tokyo, Japan \\ E-mail: pfeuty_benjamin@yahoo.fr
}

Received 3 August 2009

Accepted for publication 19 October 2009

Published 12 November 2009

Online at stacks.iop.org/PhysBio/6/046013

\begin{abstract}
A wide range of cellular processes require molecular regulatory pathways to convert a graded signal into a discrete response. One prevalent switching mechanism relies on the coexistence of two stable states (bistability) caused by positive feedback regulations. Intriguingly, positive feedback is often supplemented with negative feedback, raising the question of whether and how these two types of feedback can cooperate to control discrete cellular responses. To address this issue, we formulate a canonical model of a protein-protein interaction network and analyze the dynamics of a prototypical two-component circuit. The appropriate combination of negative and positive feedback loops can bring a bistable circuit close to the oscillatory regime. Notably, sharply activated negative feedback can give rise to a bistable regime wherein two stable fixed points coexist and may collide pairwise with two saddle points. This specific type of bistability is found to allow for separate and flexible control of switch-on and switch-off events, for example (i) to combine fast and reversible transitions, (ii) to enable transient switching responses and (iii) to display tunable noise-induced transition rates. Finally, we discuss the relevance of such bistable switching behavior, and the circuit topologies considered, to specific biological processes such as adaptive metabolic responses, stochastic fate decisions and cell-cycle transitions. Taken together, our results suggest an efficient mechanism by which positive and negative feedback loops cooperate to drive the flexible and multifaceted switching behaviors arising in biological systems.
\end{abstract}

\section{Introduction}

An essential attribute of living cells is their capacity to switch between distinct states in response to external or internal signals. Such switching behavior arises in various cellular processes such as metabolic response [1], cell differentiation [2] or cell-cycle transitions [3]. The most prevalent switching mechanism in living cells relies on the coexistence of multiple stable steady states under the same cellular conditions [4-6]. Then, specific chemical stimuli can trigger discrete transitions from one stable state to another. A necessary condition for multistability in a dynamical system is the existence of positive loops in its Jacobian system [7, 8]. Indeed, positive feedback loops have been documented in many cellular regulatory pathways, where they have been ascribed the crucial task of inducing discrete, fast and eventually irreversible cellular transitions [9-11]. However, the occurrence of multistability due to positive feedback comes at a cost, as cells may be trapped in suboptimal epigenetic states, especially when facing unknown, ambiguous or rapidly changing environmental conditions [12-14]. To overcome such a drawback, it has been suggested that negative feedback regulation could be combined and cooperate with positive feedback to facilitate escape from stable states [15-17]. As a matter of fact, positive and negative feedback loops are combined in many disparate signaling and regulatory pathways involved into discrete cellular responses [17-27], which raises the question to what extent negative feedback can complement or refine the switching properties of positive-feedback-based cellular pathways. 
Theoretical analysis of chemical or biochemical systems combining positive and negative feedback has shown that transitions from bistability to oscillations represented by a typical cross-shaped phase diagram is a pervasive feature of these systems [28, 29]. The purpose of the present study is to establish the functional advantages for biochemical bistable systems to operate close to this transition. To address this issue, we formulate a canonical model describing the dynamics of a protein-protein interaction network and study more specifically the dynamics of two-component circuits, which are able to minimally incorporate both positive and negative feedback loops. The results are organized in two parts. The first part recapitulates the basic requirements for negative feedback to convert bistability into oscillations in two-component biochemical circuits. Notably, we delineate the case where combined negative and positive feedback gives rise to a specific type of bistability where the two stable equilibria coexist and may collide pairwise with two saddle equilibria on an invariant circle. The second part focuses on the functional switching properties with respect to the control of hysteresis width, transition speed, transient-state dynamics, or stochastic transition rates, which are the characteristics of such type of bistability based on the combination of positive and negative feedback, but are not possible in the conventional mechanism of switching based on positive feedback alone. We close the paper by discussing the implications of the switching properties and the circuit topologies considered in this study, in a variety of cellular pathways and biological processes.

\section{Model}

We consider a canonical model of protein regulatory circuitry in which interactions are mediated by post-translational or transcriptional mechanisms. Under some assumptions detailed in appendix A, the dynamics of the transcriptional or post-translational circuit can be expressed in a mathematical form as a set of dimensionless differential equations:

$$
\tau_{i} \frac{\mathrm{d} x_{i}}{\mathrm{~d} \tau}=\alpha_{i}\left(1-x_{i}\right)-\beta_{i} x_{i}+\sigma_{i} \zeta_{i}(\tau)
$$

where $\alpha_{i}$ and $\beta_{i}$ are the activation and inactivation rates of the protein species $i$, which depend on the activity levels of other protein species $j$ and signaling protein species $S$ :

$$
\begin{gathered}
\alpha_{i}=a_{i}+b_{i} S^{\gamma_{1}}+\sum_{j} W_{i j}^{E} x_{j}^{\gamma_{2}} \\
\beta_{i}=1+c_{i} S^{\gamma_{1}}+\sum_{j} W_{i j}^{I} x_{j}^{\gamma_{2}}
\end{gathered}
$$

where $\gamma_{1}$ and $\gamma_{2}$ denote the degree of cooperativity within the interactions between different protein species. Other parameters have been normalized to the spontaneous inactivation rate $\left(\beta_{i}^{0}\right)$.

\subsection{Feedback loops}

The elements $m_{i j}$ of the Jacobian matrix of (1) quantify how much variations of the protein species $j$ influence the time derivative of the protein species $i$. Assuming in our model that the protein can be either monotonously activated or monotonously inhibited by other proteins $\left(W_{i j}^{E} \neq 0\right.$ implying $W_{i j}^{I}=0$ and reciprocally), the non-diagonal elements $m_{i j}$ have a definite sign. A Jacobian matrix that displays a sequence of $k$ nonzero elements $m_{i j}$ of definite sign, in which its $i$ row and $j$ column indices are circular permutations of each other, is said to display a feedback loop of size $k$, negative or positive according to the sign of the product of these elements. Diagonal elements of the Jacobian matrix incorporate an obvious negative contribution due to the spontaneous protein degradation/inactivation that can be supplemented with a positive term defining a positive feedback (self-)loop.

\subsection{Noise}

The last term on the right-hand side of equation (1) represents a source of intrinsic noise. If we assume that the number of molecules is relatively large and biochemical reactions take place in time as a Poisson process, then we can use the chemical Langevin equation [30]: $\zeta(t)$ is a white noise process of variance $\sigma_{i}^{2}$ that depends on the biochemical reaction rates (see appendix A).

\subsection{Parameters}

Throughout the paper, some parameters have been set to fixed value: $\gamma_{1}=1, \gamma_{2}=2, a_{i}=0$ and $c_{i}=0$, while other parameter values may change and are specified in the figure captions. We focus more specifically on the dynamics of post-translational circuits. Nonetheless, we further check the validity of our conclusions in the case of transcriptional circuits.

\section{Results}

\subsection{Distinct scenarios for the transition from bistability to oscillations}

Feedback loops consist of circular regulatory cascades. The sign of the loop (positive or negative) depends on the number (even or odd) of negative regulations in the loop. To begin, we consider the simplest biochemical circuit that incorporates a positive feedback loop: a single protein activating its own activity. Figure 1 shows that bistability, wherein two stable states of low- and high- $x_{1}$-activity levels coexist, emerges as the strength of the positive feedback increases. Bistability occurs for signal values ranging between $S_{1}$ and $S_{2}$ at which two, stable and unstable, equilibria appear or disappear through a saddle-node bifurcation. The two branches of a saddle-node bifurcation meet at a cusp-bifurcation point where the amplitude of the switch and the size of the bistability region decrease to zero $\left(W_{11}^{E} \approx 3.4\right)$. These basic properties of a bistable switching behavior are shared by any system that incorporates a single positive feedback loop comprising eventually several regulatory links and components (e.g. mutual activation or inactivation).

We analyze further how the presence of negative feedback modulates such conventional switching behavior based on 
(a)
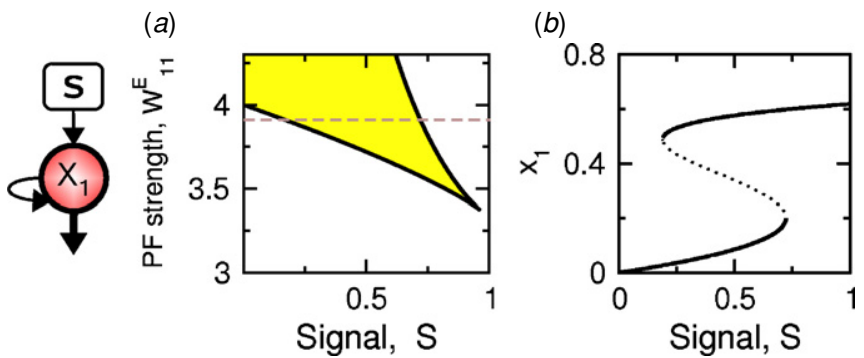

Figure 1. Conventional bistable switch based on a single positive feedback loop. Steady-state properties of a single-component circuit as a function of the signal strength and self-activation level that measures positive feedback (PF) strength. Other parameters: $b_{1}=0.13$. (a) Phase diagram where the white domain corresponds to monostability (one stable fixed point) and the yellow domain corresponds to bistability (two stable and one unstable fixed points). The solid line indicates a saddle-node bifurcation. $(b)$ Bifurcation diagram (the solid lines indicate stable fixed points, the dotted line indicates unstable fixed points) for $W_{11}^{E}=3.9$ (dashed line of $(a)$ ). a single positive feedback loop. To this end, the simplest approach is to consider a two-component circuit where the selfactivating $x_{1}$ component, called master, behaves like a bistable switch $\left(W_{11}^{E}=3.9\right)$ and is interacting with a $x_{2}$ component, called partner, establishing a negative feedback loop in two possible manners as depicted in figure 2.

In a first circuit topology, the master component both activates and is inhibited by the partner component (figures $2(a)-(c)$ ). For such a circuit, we define a control parameter $Q_{1}$ that increases with the inhibitory regulation, $W_{12}^{I}$, and decreases with the signal sensitivity of the master component, $b_{1}$. $Q_{1}$ measures the strength of negative feedback, with $Q_{1}=0$ indicating an absence of negative feedback. Increasing the negative feedback strength can affect positive feedback-based bistability in three typical manners. First, for a partner dynamics not too slow or not too much selfactivating, the increase of $Q_{1}$ reduces the bistable domain and two stable fixed points collide through a cusp (pitchfork) (a)

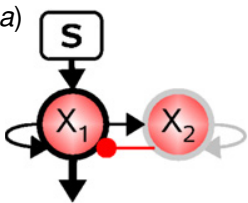

(b)

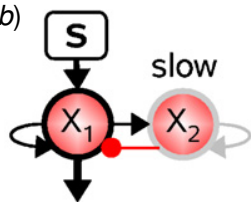

(c)

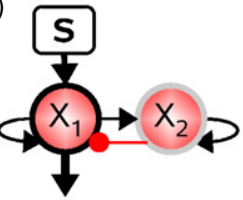

(d)

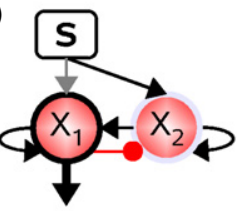

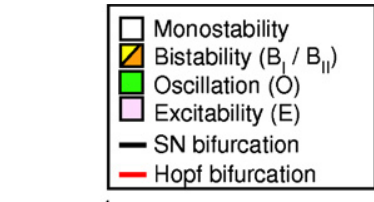
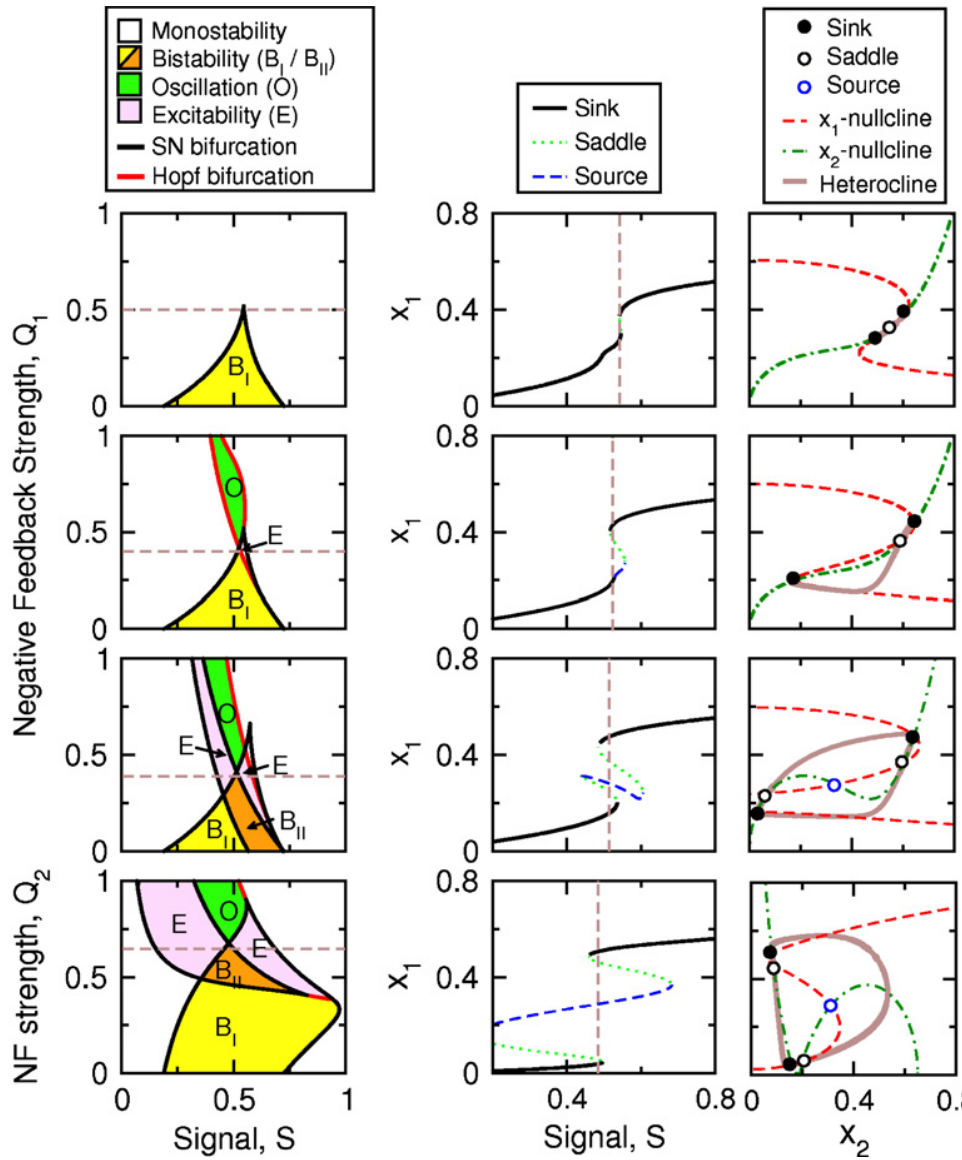
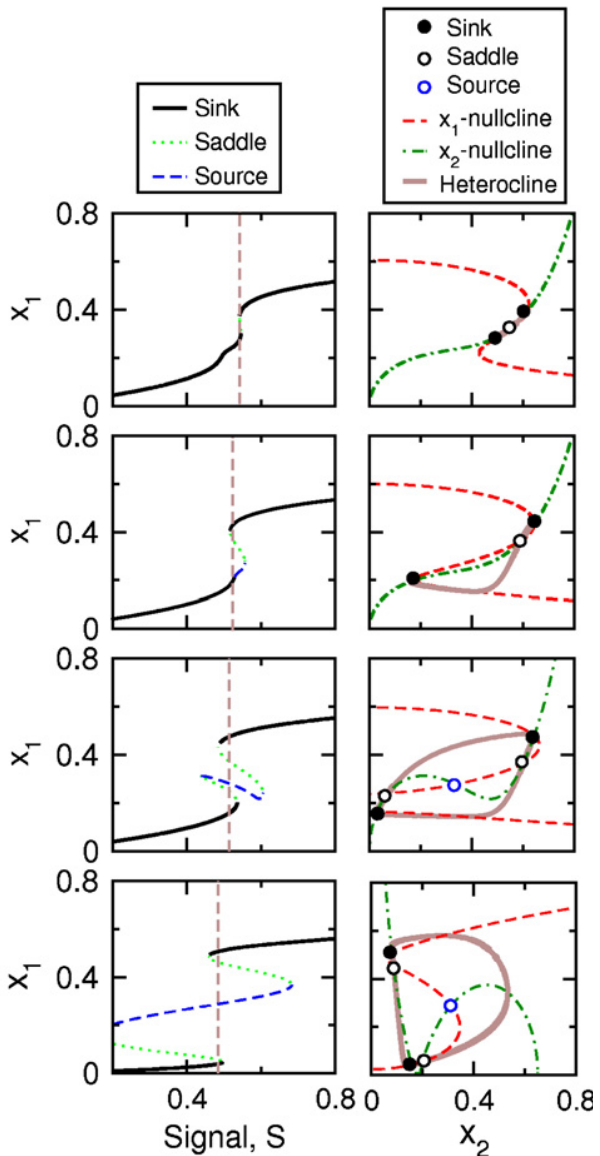

Figure 2. Transitions from bistability to oscillations in the presence of negative feedback. Phase diagram, bifurcation diagram and phase plane associated with different topologies and parameters of a two-component circuit: $(a) \tau_{1}=1, \tau_{2}=1, W_{11}^{E}=3.9, W_{22}^{E}=3$, $b_{1}=0.13\left(1+Q_{1}\right), b_{2}=0, W_{12}^{I}=0.5 Q_{1}, W_{21}^{E}=3$. (b) Same as $(a)$ except $\tau_{2}=10$. $(c)$ Same as $(a)$ except $W_{22}^{E}=3.8$ and $W_{21}^{E}=1$. (d) Same as $(a)$ except $W_{22}^{E}=4.2, b_{1}=0.13\left(1-Q_{2}\right), b_{2}=0.26 Q_{2}, W_{21}^{I}=1, W_{12}^{E}=0.6$. Bifurcation diagrams are shown for the value of $Q_{i}$ indicated by the dashed line on the phase diagram. Phase planes are shown for the value of $S$ indicated by the dashed line on the bifurcation diagram. All legends associated with phase diagram, bifurcation diagram and phase plane are shown on the top of the figure. Bifurcation and phase diagrams are all based on the linear stability analysis of fixed points (see appendix B). Monostability: one sink; type-I bistability $\left(B_{I}\right)$ : two sinks and one saddle; excitability $(E)$ : one sink, one saddle and one source; type-II bistability $\left(B_{I I}\right)$ : two sinks, two saddles and one source; oscillation $(O)$ : one source. 
bifurcation (figure $2(a)$ ), similar to the case where positive feedback strength is reduced (figure 1). The presence of the negative feedback merely counteracts the effect of the positive feedback. Second, for a delayed negative feedback due to a slow partner dynamics (e.g. $\tau_{2}=10$ ), increase of $Q_{1}$ also reduces the bistable domain, but can additionally give rise to oscillatory or excitable regime (figure $2(b)$ ). In this case, the two stable fixed points are destabilized through a saddle-node bifurcation and a Hopf bifurcation respectively. Third, for higher partner self-activation, increase of $Q_{1}$ not only generates oscillations or excitability but also produces a specific type of bistability where two saddle equilibria are present instead of a single one and collide with stable fixed points (figure 2(c)). In the following, such bistability associated with two saddle points is called type-II while type-I refers to the existence of a single saddle point. These three typical ways by which negative feedback affects bistability can be captured by simple theoretical considerations described in appendix B. It is confirmed in two-component circuits that negative feedback must operate at enough slow time scale or/and must be mediated by a sharply activated component to allow that two stable fixed points of a bistable system can be destabilized simultaneously while being far from each other, giving rise to a transition from a bistable to an oscillatory regime.

Type-II bistability can also occur in an alternative feedback architecture where the self-activating master component is activated by and inhibits the partner component (figure $2(d)$ ). The strength of the negative feedback is now measured by the parameter $Q_{2}$ associated with the increase in $b_{2}$ and the decrease in $b_{1}$. Like the previous topology, type-II bistability requires strong enough negative feedback and selfactivation of the partner component. In fact figures $2(c)$ and (d) correspond to two switching scenarios associated with two distinct domains in the parameter space, which differ primarily by the level of negative regulation within the negative feedback loops. Figure 3 depicts clearly the difference between those two cases of type-II bistability where the signal-induced activation of a first protein activates a second protein, which in turn can (i) slightly reduce the first protein's activity or (ii) turn it off, respectively. In one case, the partner contributes to the switch-off event only; while in the other case, the partner contributes to the switch-on event only. Thus, despite their differences, both type-II bistable switching scenarios are characterized by a separate and independent control of switchon and switch-off events.

\subsection{Functional switching properties associated with type-II bistability}

In the previous section, we focused on the emergence of a specific type of bistability, called type-II, in the presence of the appropriate combination of positive and negative feedback. What are the specific switching properties of type-II bistable system as compared with the conventional case of bistability based on positive feedback alone? Could these switching properties provide functional advantages in some physiological contexts? In the following, we study several
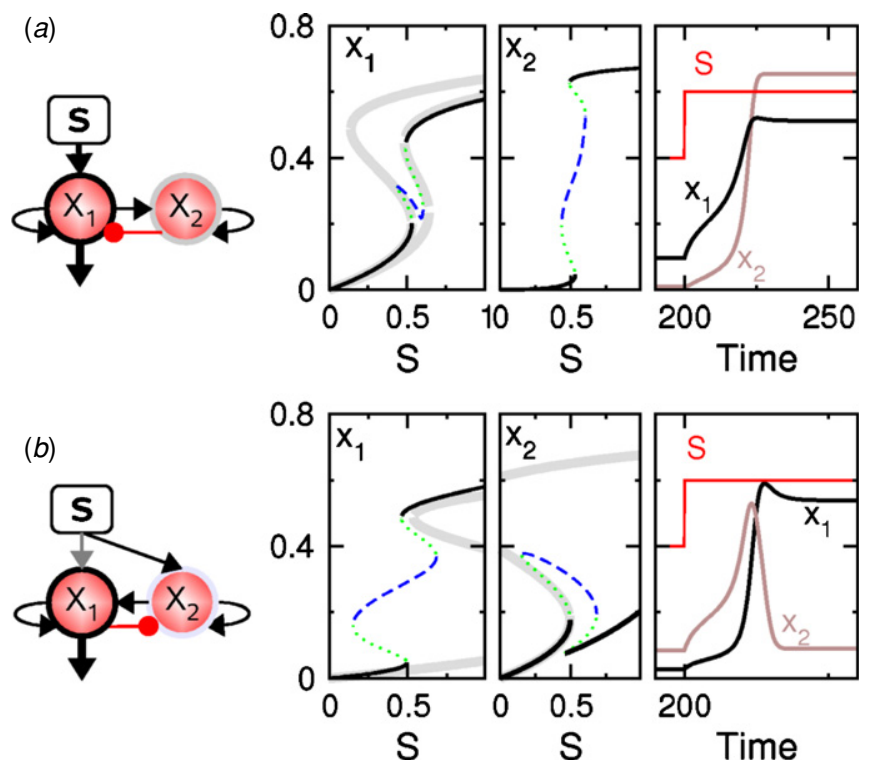

Figure 3. Two main switching scenarios associated with type-II bistability. (a) Same motif and parameters as in figure 2(c). The signal-driven master $x_{1}$ component activates its partner inhibitor. The corresponding bifurcation diagram (left panel) is compared with the two steady-state curves (gray thick line) associated with the cases where the master is either not inhibited or constantly inhibited. Time evolution of $x_{1}$ and $x_{2}$ activities in response to a signal step (right panel) indicates that both $x_{1}$ and $x_{2}$ activities switch-on. (b) Same motif and parameters as in figure 2(d). The master $x_{1}$ component inhibits its signal-driven partner activator. The corresponding bifurcation diagram (left panel) is compared with the two steady-state curves (gray thick line) associated with the single-master-component dynamics or single-partner-component dynamics. The temporal response to a signal step indicates that only $x_{1}$ activity switches permanently while $x_{2}$ activity switches transiently.

specific switching properties as the response time, the quasistationnarity or the effect of noise.

3.2.1. Switching response speed. As it is essential for cells to rapidly and efficiently adapt in response to environmental changes, protein regulatory circuits must have evolved mechanisms to produce prompt switching behavior.

We first define the switching speed in a bistable system as the speed to transit from the neighborhood of a saddlenode bifurcation point to the neighborhood of the other, stable, steady state (see appendix D). Of course, the switching speed depends on the intrinsic time scales of the system dynamics (i.e. $\tau_{i}$ in our model) associated with the time constants of the biochemical processes involved in protein synthesis, degradation or post-translational modification. However, it also depends on the feedback structure of the circuit. Figure 4 shows how the interplay between positive and negative feedback affects the switching speed. Increasing the positive feedback strength accelerates significantly the switching speed (figure 4(a)) together with the hysteresis width (figure $4(b)$ ) such as to produce a fast and irreversible transition. The addition of negative feedback can decorrelate these two properties, by restoring a reversible switching behavior while preserving the fast switching response. As a result, among 

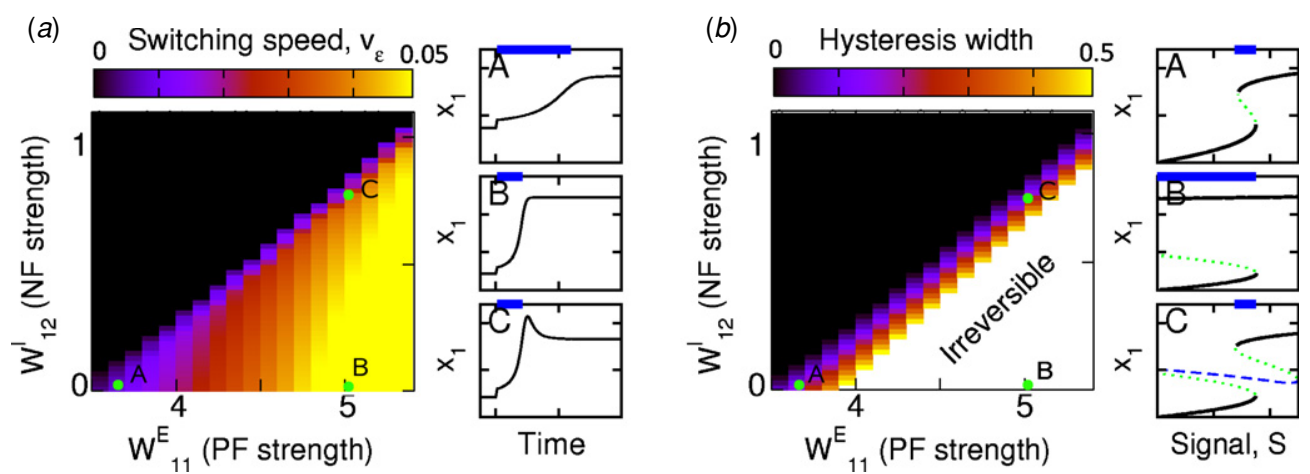

Figure 4. Fast and reversible transitions in type-II bistable systems. The switching speed (equation (D.3) with $\epsilon=0.04)(a)$ and hysteresis width $(b)$ of a two-component circuit (same motif as figure $2(c)$ ) as a function of positive and negative feedback strength $\left(W_{11}^{E}\right.$ and $W_{12}^{I}$ respectively). Black domains are associated with a non-bistable regime. Panels of $(a)$ show example of time evolution of $x_{1}$ activity in response to a small perturbation near the bifurcation point, from which switching speed is computed. Panels of $(b)$ show bifurcation diagram from which hysteresis width is computed. Fixed parameters: $\tau_{1}=1, \tau_{2}=1, W_{22}^{E}=3.8, W_{21}^{E}=1, b_{2}=0 . b_{1}$ is adjusted to keep constant the signal threshold associated with the switch-on event while $W_{11}^{E}$ is varied. Panel A: $W_{11}^{E}=3.6, W_{12}^{I}=0$; panel $\mathrm{B}: W_{11}^{E}=5, W_{12}^{I}=0$; panel C: $W_{11}^{E}=5, W_{12}^{I}=0.75$.

two circuits with similar switching and hysteresis (panels A and $\mathrm{C}$ of figure $4(b)$ ) amplitudes, the type-II bistable system combining positive and negative feedback exhibits a much faster response than the type-I bistable system without negative feedback (panels A and C of figure 4(a)). Alternatively, among two circuits with similar switching speed (panels B and $\mathrm{C}$ of figure $4(a)$ ), only the type-II bistable system can display a reversible switching behavior.

These results clearly show how type-II bistable systems benefit from complementary roles between positive and negative feedback wherein the former controls the switching speed (and amplitude) and the latter controls the extent of hysteresis (i.e. the degree of irreversibility), allowing both fast and reversible state transitions.

\subsubsection{Transient-state dynamics. Many types of cellular} behavioral changes are transient, such as during the celldivision cycle or certain types of differentiation. In these processes, individual cells, some time after a step or pulse of signal triggered a transition to a specific state, switch back to the original state or to a further different state. Thus, the main feature of a transient state is that its termination is caused by the internal dynamics rather than an external control.

Here, we show that type-II bistable switches can naturally generate such transient states in response to both pulse or step of signal. One basic paradigm of transient-state dynamics is related to the existence of an excitable dynamical regime where a perturbation beyond a threshold value gives rise to a large non-monotonous response, eventually to a state close to being stable (sometimes called an attractor ruin) before returning to the original steady state. For a standard type-II bistable switch (figure 5(a)), a signal pulse can induce a transient state (panel $\mathrm{C}$ of figure $5(c)$ and figure $5(d)$ ) in the excitable regime, which contrasts with the permanent switching response (panel $\mathrm{C}$ of figure $5(c)$ ) in the bistable regime or the rapid monotonic relaxation (panel A of figure 5(c)) for not excitable enough system or not strong enough perturbation. Transient states can also occur in the bistable regime of a type-II bistable switch (figures $5(b)$ and $(e)$ ), where the switch from one state to another is associated with a large excursion in the phase space during which one variable is transiently activated (switched on and off). In this case, a step of signal can induce a transient state (panel E of figure 5(c)). In both excitable or bistable regime, the duration of transient states is determined by intrinsic time scales as well as the degree of instability of the attractor ruin.

For the reasons above, a suitable combination of positive and negative feedback makes type-II bistable systems capable to generate transient states with various properties: transient states can (i) display variable durations, (ii) be elicited by a pulse or a step of signal, (iii) be followed by the return to the original state or a switch to a further different state.

3.2.3. Stochastic switching dynamics. The discreteness of molecule number and of biochemical reactions leads to temporal and spatial fluctuations in protein concentrations. Such intrinsic noise can induce stochastic transitions between the two stable states of a bistable biochemical switch.

We investigate how type-II bistable switches (same motifs and parameters as in figure $2(d)$ ) behave in the presence of intrinsic noise (equations (1) and (A.2)) where we define $\tilde{\sigma}_{i}=\sqrt{\tau_{i}} / \sqrt{N_{i}}$ and $\tilde{\sigma}=\tilde{\sigma}_{1}=\tilde{\sigma}_{2}$. Figure 6(a) depicts how the transition rate depends on the intrinsic noise level, $\tilde{\sigma}$, and the negative feedback strength, $Q_{2}$. The signal value is adjusted such that the circuit state switches on and off with the same probability. In the absence of noise, transitions occur only for $Q_{2}>0.69$, in the oscillatory regime characterized by the periodic switch-like alternation between low- and high- $x_{1}$ activity. In the presence of noise, the transition rate increases significantly when $Q_{2}$ increases within the type-II bistable regime. The level of negative feedback can therefore modulate the transition rate of a bistable switch over a wide range, about tenfold for $\tilde{\sigma}_{i}=0.05$, giving rise to either very robust or very sensitive switching behavior. The inflexion point occurs when phase space trajectories follow different paths during switch-on and switch-off transitions, respectively. For 
(a)

(b)

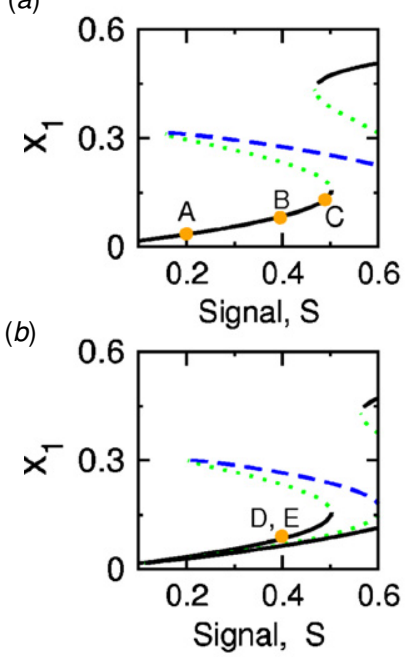

$(c)$

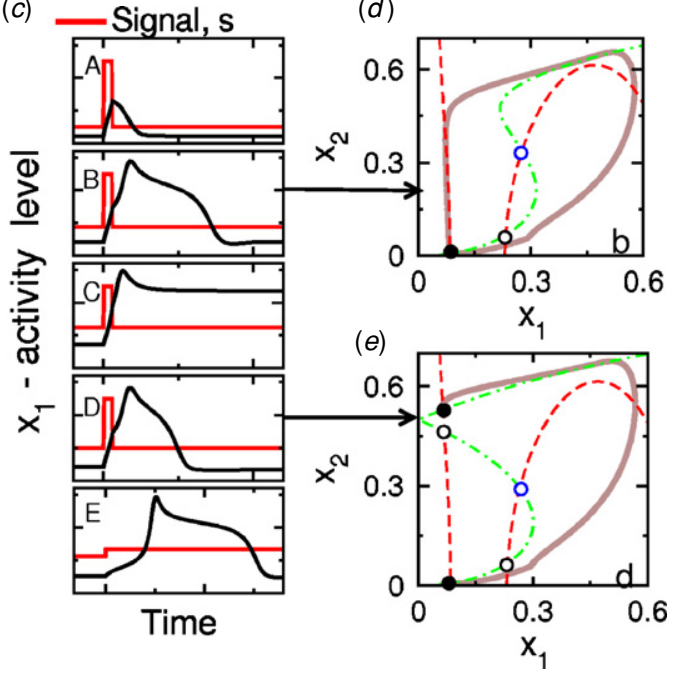

Figure 5. Transient states in type-II bistable systems. (a) Bifurcation diagram (see legend in figure 2) of a two-component circuit (same motif as figure $2(c)$ ) with parameters $\tau_{1}=1, \tau_{2}=1, W_{11}^{E}=4.5, W_{22}^{E}=3.8, b_{1}=0.2, b_{2}=0, W_{12}^{I}=0.5, W_{21}^{E}=1(b)$ Same as A except $W_{22}^{E}=4$. (c) Time evolution of $x_{1}$ activity (black solid line) in response to a signal pulse or step (red solid line) is shown for different initial circuit state. (A: $S=0.2$, pulse; B: $S=0.4$, pulse; C: $S=0.5$, pulse; D: $S=0.4$, pulse; E: $S=0.4$, step). (d, e) $x_{1}$ activity (brown solid line) associated with panels B and D of $(c)$ are represented on the phase plane with nullclines (dashed and dash-dotted lines) and fixed points (circles); see legend in figure 2.

the type-I bistable system (e.g. $Q_{2}=0.2$ ), high levels of noise $\left(\tilde{\sigma}_{i}=0.05\right)$ barely trigger transitions between lowand high- $x_{1}$ activity (figure $6(b)$ ). In contrast, for type-II bistable systems (e.g. $Q_{2}=0.6$ ), relatively low levels of noise $\left(\tilde{\sigma}_{i}=0.02\right)$ can trigger transition between low- and high$x_{1}$ activity (figure $6(c)$ ). The sharp distribution of $x_{1}$-activity levels around the stable fixed point indicates that relatively high stochastic transition rates can arise together with long periods of low fluctuations.

In summary, the stochastic transition rate of type-II bistable circuit can be tuned by parameters (e.g. those modulating the negative feedback strength) that move the attractor basin boundaries close to or away from stable fixed points in a flexible manner.

\section{Discussion}

\subsection{The specific switching properties of type-II bistable systems}

In this paper, we investigate the switching properties of protein circuits composed of interacting positive and negative feedback loops. It is well known that adding negative feedback to positive feedback can turn bistability into oscillations in both chemical or biological systems [28, 29, 31-33]. We find that circuits operating close to this transition exhibit a flexible control of switching behavior far beyond that attributed to the conventional bistable switch based on a single positive feedback loop. This is especially the case for circuit that displays a specific type of bistability, named type-II, which provides a prototypical example of a two-dimensional system that can alternatively switch or oscillate between two states. Type-II bistable systems are able to generate transient switching responses, to display tunable transition rates and to decorrelate hysteresis size with switching amplitude and speed. Thus, such bistable systems can give rise to a great diversity and flexibility of switching behavior characterized by a wide spectrum and tunability of noise sensitivity or degree of reversibility.

These results suggest that negative and positive feedback loops can cooperate not only to tune the properties of biological oscillators [34-36], the gain of metabolic fluxes [37] or the spatial patterning [38], but also in the context of biochemical switching behaviors, requiring that these feedback loops are suitably combined. Type-II bistability arises indeed in simple circuit topologies where, typically, a master regulator is involved in a positive feedback loop and interacts with a partner regulator through a negative feedback loop. In fact, two typical architectures are possible where the partner is located either upstream or downstream to the master regulator. In any case, a sharp or discrete activation of the partner and a fast positive feedback (as compared to the speed of the negative feedback) are necessary to restrict the effect of the negative feedback to the case when the master regulator has been fully activated, allowing a separate and tunable control of switchon and switch-off events and their respective thresholds. This specific feedback architecture ensures that negative and positive feedback does not interfere and cancel their effect, but instead cooperate for flexible switching behavior as described above.

It is important to emphasize that type-II bistable systems provide an illustrative but incomplete picture of biochemical switches combining positive and negative feedback loops or/and operating near the transition to excitable or oscillatory regimes. On the one hand, biochemical circuits can involve several negative $[32,33]$ or positive [39-42] feedback loops interlocked in more complicated ways than the topologies described in the present study. On the other hand, stable steady states can loose their stability through Hopf bifurcations, 

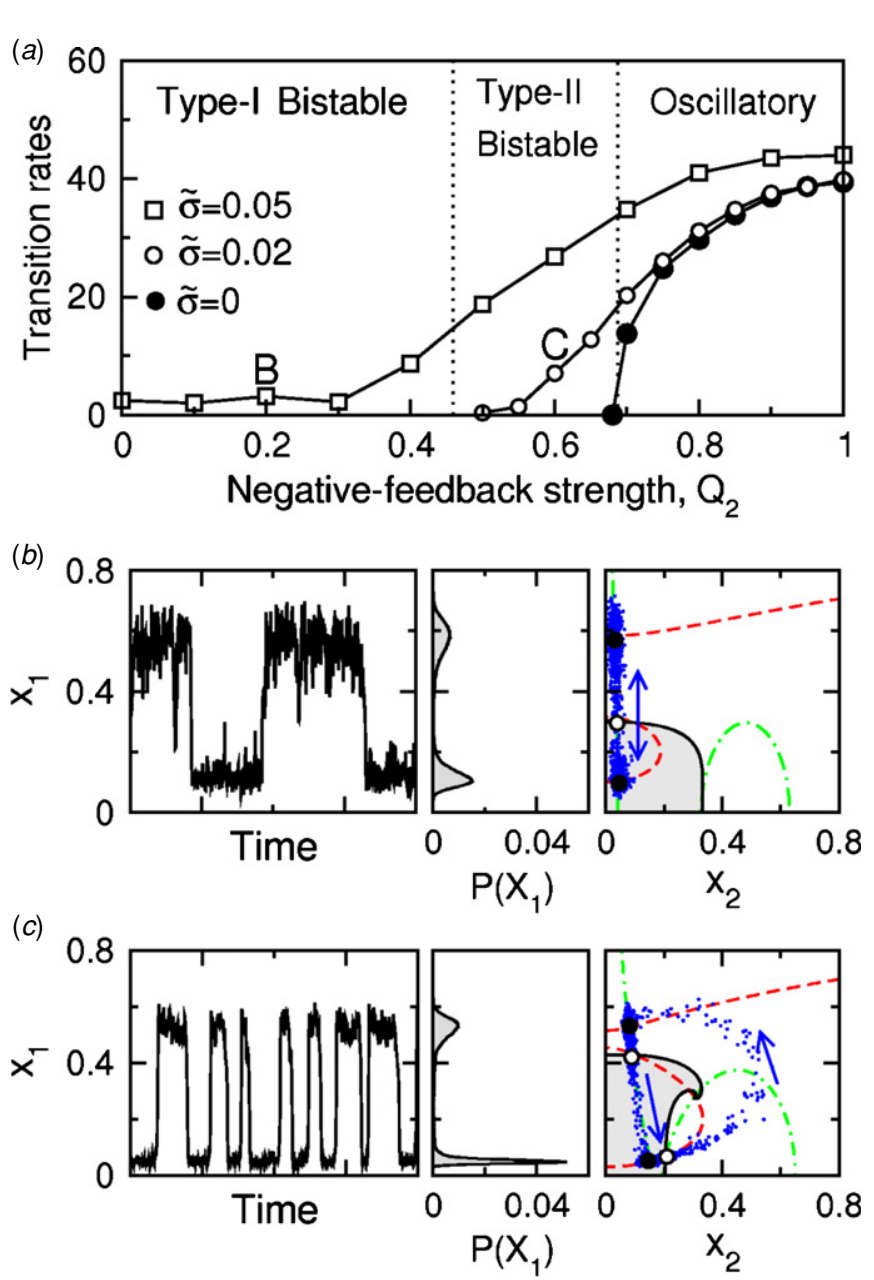

Figure 6. Tunable noise-induced transitions in type-II bistable systems. Stochastic transitions within the circuit described in figure $2(d)$ in the presence of intrinsic noise. The signal level is fixed and adjusted to have similar transition rates in both directions. (a) Transition rates as a function of negative feedback strength, $Q_{2}$. $(\mathrm{b}, \mathrm{c})$ Stochastic time evolution of $x_{1}$ activity (left), probability distribution (center) and trajectories represented in the phase plane (right). On phase planes are shown nullclines (dashed and dash-dotted lines), fixed points (circles), attractor basin (gray and white), basin boundary (solid line) and the circuit state at successive time interval (blue points). (b) For $Q_{2}=0.2$ (type-I bistability), rare transitions between $x_{1}$-active and $x_{1}$-inactive states elicited by high level of noises ( $\tilde{\sigma}=0.05$ ). (c) For $Q_{2}=0.6$ (type-II bistability), frequent transitions between $x_{1}$-active and $x_{1}$-inactive states elicited by relatively low levels of noises $(\tilde{\sigma}=0.02)$.

supercritical or subcritical [28, 32], instead of saddlenode bifurcations especially in high-dimensional systems. Nevertheless, these considerations do not preclude that highdimensional biochemical circuits exhibit type-II bistable dynamics as long as the feedback architecture and time scale properties enable a separate control of switch-off and switchon events.

Our study raises the question of whether and which cellular pathways combine positive and negative feedback such as to operate near the transition between bistable and oscillatory regimes. Among possible candidates, many signaling and regulatory pathways involved in discrete cellular responses actually combine positive and negative feedback loops (figure 7), which is a necessary though not sufficient condition to exhibit type-II bistability. In the following section, we provide some concrete examples of such cellular pathways that are likely to deploy a type-II bistable switching behavior.

\subsection{Fast, stochastic and transient switching behaviors in biological systems}

It is essential for any organism to rapidly and reversibly adapt in response to changes in nutrient availability and other environmental parameters. Regulatory mechanisms such as negative autoregulation have been shown to contribute to speeding up the reaction time of physiological responses [43]. Our study revealed a related mechanism allowing a fast and reversible switching behavior: strong positive feedback that triggers prompt and irreversible transitions is combined with negative feedback that restores reversible transitions while preserving the high transition speed. Such a mechanism may have evolved, for instance, to ensure a rapid and reversible response of yeast cells to changing levels of glucose. The yeast glucose utilization network consists of two interconnected signaling pathways (Snf3/Rgt2Rgt1 and Snf1-Mig1) incorporating multiple feedback loops $[19,44]$ (figure 7(b)). At low glucose, activation of Snf3 relieves, mainly via Std1, the Rgt1-dependent repression of HXT genes encoding glucose transporters and results in activation of the glucose transcriptional repressor Mig1. In turn, Mig1-dependent repression of Mthl contributes to the phosphorylation of Rgt1, thereby promoting the expression of the HXT1 gene and giving rise to a positive feedback loop required to maintain high Mig1 levels. At the same time, Mig1-dependent repression of Mig 1, Snf3, HXT2-4 genes contributes to multiple negative feedback loops. This feedback topology wherein a starter component (i.e. Snf3/HXT2-4) contributes to the activation of the master regulator (Mig1), which further inactivates the starter component, is likely, according to our findings, to enable a prompt induction and repression of the glucose utilization pathways when glucose is either exhausted or available, respectively.

Noise-induced cellular transitions can also play an active role into biological adaptation by promoting phenotypic heterogeneity in a clonal cell population and subsequently allowing a subset of cells to adapt more effectively to harmful environmental changes [12,45]. Accordingly, cells are likely to evolve their inter-phenotype switching rates according to the frequency of environmental changes [46], which can be done by tuning their intrinsic noise or their switching sensitivity to noise. For the latter mechanism, our study proposed that the presence of negative feedback in addition to positive one allows tunable control of stochastic switching rates. Interestingly, cell fate decisions that are influenced by stochastic processes are often associated with regulatory networks combining positive and negative feedback such as those involved in the competence or sporulation processes of Bacillus subtilis [16, 47, 48] (figures 7(a) and $(h)$ ). A role for type-II bistable switching in these processes is indeed 

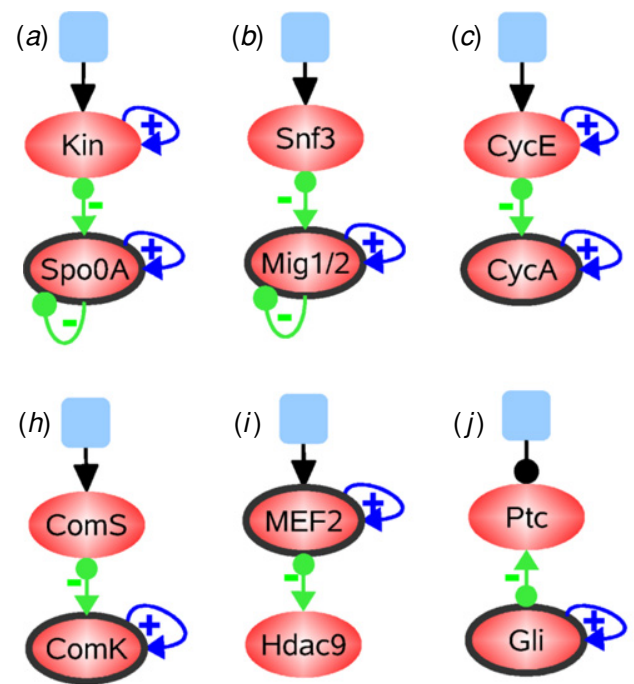
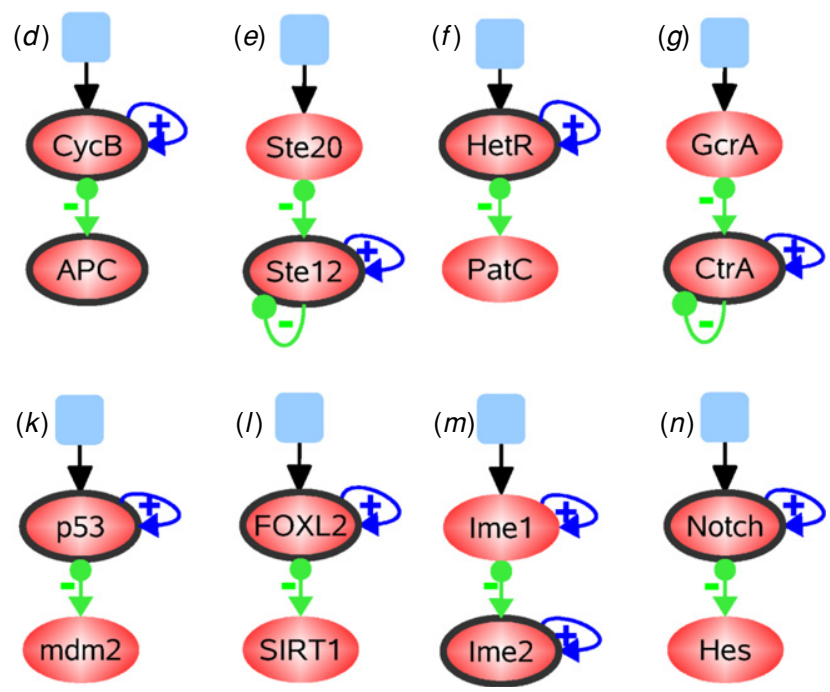

Figure 7. Examples of cellular pathways combining positive and negative feedback loops. Two-component circuits as a schematic representation of signaling or regulatory pathways combining positive feedback loop (blue, + ) and negative feedback loop (green, - ). The solid circle denotes a master regulator that is involved into $(a)$ sporulation initiation in Bacillus subtilis [18]; $(b)$ glucose utilization in budding yeast [19, 44]; (c) S-phase initiation in the cell-division cycle of eukaryotes [49]; $(d)$ mitotic control in the cell-division cycle of eukaryotes [49]; $(e)$ pheromone response in budding yeast [22]; $(f)$ heterocyst differentiation in cyanobacteria [23]; $(g)$ cell constriction initiation in caulobacter [25]; $(h)$ competence induction in Bacillus subtilis [18]; $(i)$ ovarian differentiation in mammals [27]; $(j)$ differentiation in ventral neural tube [20]; $(k)$ p53-dependent stress response in mammals [21]; $(l)$ muscle differentiation [24]; $(m)$ meiosis initiation in budding yeast [26]; $(n)$ notch-dependent differentiation [33].

supported by a study by Süel and co-workers in which a model of the competence regulatory circuit displays a type-II bistable regime for a certain range of parameters (see the supporting material of [16]).

Competence and sporulation, besides being influenced by noise, are temporally constrained cellular processes for which the cell adopts a transient state, or a sequence of transient states, to further come back to the original state. Another well-documented example of transient switching behavior is provided by the cell-division cycle of which the ordered succession of stages are controlled by the sequential activation of cyclin-dependent protein kinases (Cdks) [49]. Interestingly, these key regulators are immersed in a regulatory network that combines multiple feedback loops [49] (figures 7(c) and $(d)$ ). Positive loops mediated by mutual inhibition with Cdk inhibitors or mutual activation with transcription factors contribute to switching onto Cdk-active states. In turn, negative loops ensure further inactivation of Cdks (e.g. Cdk1 activates the anaphase-promoting complex, which leads to cyclin destruction and $\mathrm{Cdk} 1$ inactivation). Our study suggests that such feedback architecture, able to give rise to type-II bistability, accounts for both clock-like and switch-like properties of cell-cycle progression depicted as a cyclical sequence of transient states, which can eventually be turned into stable states if checkpoint conditions are not satisfied. This interpretation is in agreement with those postulated in previous modeling studies $[50,51]$, which have described the directional cell-cycle progression monitored through checkpoint mechanisms by a dynamic structure where saddle-node bifurcations arise on an invariant circle, similar to type-II bistable systems.

\section{Conclusion}

The frequent combination of positive and negative feedback loops in biological systems suggests that this type of circuits possess some performance advantage not only for spatial pattern formation or oscillatory behavior but also for switching behavior. Strong positive feedback is necessary to produce prompt and robust switches, but may also induce irreversible transitions and lead to excessively stable states, thereby impairing the adaptive behavior of the cell. Our study posits that the suitable addition of negative feedback can allow a selective and contextual destabilization of these states while preserving the switch-like behavior triggered by positive feedback. During a developmental cycle as the cell-division or sporulation cycle, such a cooperative mechanism would contribute to the cellular progression along a cyclical sequence of states, eventually controlled by checkpoints. In many other cellular processes, the same mechanism would instead promote fast and reversible signal-driven adaptive transitions. Alternatively, it could also enable an efficient noise-driven exploration of the phenotypic space, which is crucial for the evolutionary adaptation of cells that must anticipate unforeseen environments or adapt to unknown challenges.

\section{Appendix A. Derivation of the canonical model for transcriptional and post-translational networks}

We have formulated a set of dimensionless differential equations (equation (1)) to describe the dynamics of protein networks. In the following, we give the details of the assumptions and renormalizations that were made to derive these equations. 
First, we consider the case of protein species $i$ (with $N_{i}$ copy number) regulated at the post-translational level by phosphorylation and dephosphorylation mechanisms. Assuming a fast catalytic reaction rate compared to the binding rate and using the law of mass action, we can write the following equation for the time evolution of the number of activated proteins:

$$
\frac{\mathrm{d} X_{i}^{+}}{\mathrm{d} t}=\tilde{\alpha}_{i}\left(N_{i}-X_{i}^{+}\right)-\tilde{\beta}_{i} X_{i}^{+}+\eta(t)
$$

where $\tilde{\alpha}$ and $\tilde{\beta}$ are the activation and inactivation rates, and $\eta$ is a white noise term. Equation (1) can be derived from equation (A.1) with $\tau=\mathrm{d}_{1} t, x_{i}=X_{i}^{+} / N_{i}, \tau_{i}=\mathrm{d}_{1} / d_{i}\left(d_{i}\right.$ being the spontaneous inactivation rate of protein species $i$ ). The variance $\sigma_{i}^{2}$ is given by

$$
\sigma_{i}^{2}=\left(\alpha_{i}\left(1-x_{i}\right)+\beta_{i} x_{i}\right) \frac{\tau_{i}}{N_{i}} .
$$

In the case of proteins regulated at the transcriptional level, one can also derive equation (1). Let us consider two classes of transcriptional factors, inducers $X_{j}$ and repressors $Y_{j}$, that can bind and unbind at some rate $k_{i j}^{+}, k_{i j}^{-}, K_{i j}^{+}, K_{i j}^{-}$ with a cooperativity $n$, to a specific DNA-binding site of the gene encoding the protein species $i$. Given a constant total DNA-binding site concentration and averaging over many fast binding and unbinding events, the rate of mRNA production or promoter activity, $p_{i}$, is given by the probability that the site is bound by an inducer, times the maximal transcription rate, $k_{m}[52]$ :

$$
p_{i}=k_{m} \frac{\sum_{j} k_{i j}^{+} / k_{i j}^{-} X_{j}^{n}}{1+\sum_{j} k_{i j}^{+} / k_{i j}^{-} X_{j}^{n}+\sum_{j} K_{i j}^{+} / K_{i j}^{-} Y_{j}^{n}} .
$$

For sufficiently slow degradation of proteins as compared to mRNA, a quasi-steady state approximation can be used and leads to the following equation for the time evolution of protein concentrations $X_{i}$ :

$$
\frac{\mathrm{d} X_{i}}{\mathrm{~d} t}=\gamma_{+} p_{i} / q_{m}-\gamma_{-} X_{i}+\eta(t)
$$

with $q_{m}, \gamma_{+}$and $\gamma_{-}$are the mRNA degradation rate and the protein translation and degradation rates, respectively. Equations (1), (2) and (3) can be derived from equations (A.3) and (A.4) after defining suitable rescaled variables and parameters: $\tau=\gamma_{-} t, \lambda=\frac{k_{m} \gamma_{+}}{q_{m} \gamma_{-}}, x_{i}=X_{i} / \lambda$, $W_{i j}^{E}=\lambda^{n} k_{j}^{+} /\left(d_{i} k_{j}^{-}\right), W_{i j}^{I}=\lambda^{n} K_{j}^{+} /\left(d_{i} K_{j}^{-}\right), \tau_{i}=\left(\alpha_{i}+\beta_{i}\right)$. Finally, the Langevin noise associated with stochastic protein translation and degradation events is given by

$$
\sigma_{i}^{2}=\left(\alpha_{i}\left(1+x_{i}\right)+\beta_{i} x_{i}\right) \frac{\tau_{i}}{\lambda} .
$$

It is important to mention that for both transcriptional and post-translational regulation, we assume that the binding/unbinding events (between transcription factors and DNA-binding sites or between enzymes and proteins) occur independently, omitting the case of cross-regulatory events. We also neglect the case where proteins are regulated at the level of mRNA or protein degradation. Cross-regulations as well as regulations at multiple levels are expected to provide additional sources of non-linearities and feedback mechanisms that would certainly contribute to enrich and refine the dynamics of protein-protein interaction network.

\section{Appendix B. Linear stability analysis of a two-component circuit}

For two-dimensional flows, attractors can be only a fixed point or a limit cycle. The type of fixed point can be determined by the sign of the trace and determinant of the Jacobian matrix. Because the trajectories of the dynamical systems (equation (1)) are bounded into the trapping subdomain ]0, 1[ of the phase space, the Poincare-Bendixon theorem can be applied to identify the presence of a limit cycle associated with the occurrence of a single unstable equilibrium. The Poincare index theory can also be applied: if all fixed points are hyperbolic, then there must be an odd number $2 n+1$ of equilibria of which $n$ are saddles and $n+1$ are sinks or sources. Bistable systems therefore possess $n$ saddles and $n-1$ sources, with $n=1$ and $n=2$ being associated with type-I and type-II bistability, respectively.

\section{Appendix C. Typical effects of negative feedback on bistability}

In order to characterize schematically the manifold influences of negative feedback on bistability, we depart from the original model (equation (1)) by assuming that the first component, which can exhibit bistability $\left(W_{11}^{E}>3.4\right)$, activates its own inhibitor according to an arbitrary function $G$ :

$$
\left\{\begin{aligned}
\tau_{1} \mathrm{~d} x_{1} / \mathrm{d} t & =\left(a_{1} S+W_{11}^{E} x_{1}^{2}\right)\left(1-x_{1}\right)-\left(1+W_{12}^{I} x_{2}^{2}\right) x_{1} \\
& =F\left(S, x_{1}, x_{2}\right) \\
\tau_{2} \mathrm{~d} x_{2} / \mathrm{d} t & =G\left(x_{1}\right)-x_{2} .
\end{aligned}\right.
$$

We consider separately the effect of constant, linear and nonlinear contributions of that activation function $G$ :

$$
G\left(x_{1}\right)= \begin{cases}c_{0} & \in] 0,1[ \\ c_{0} x_{1} & \in] 0,1[ \\ c_{0} H\left(x_{1}-x_{0}\right) & \in] 0,1[.\end{cases}
$$

A constant activation of the negative regulation has a straightforward effect of renormalizing the dynamics of the first component:

$$
\frac{\tau_{1}}{1+Q} \frac{\mathrm{d} x_{1}}{\mathrm{~d} t}=\left(\frac{a_{1}}{1+Q} S+\frac{W_{11}^{E}}{1+Q} x_{1}^{2}\right)\left(1-x_{1}\right)-x_{1}
$$

where $Q=W_{12}^{I} c_{0}^{2}$, meaning that increasing the negative feedback strength has a similar effect as decreasing the selfactivation (i.e. the positive feedback strength), together with the signal sensitivity and the time scale.

A linear activation of the negative regulation can give rise to a scenario where fixed point is destabilized through a Hopf bifurcation. The crossing of the imaginary axes by a conjugated pair of eigenvalues occurs if $Q$ satisfies both these inequalities for the steady state $x_{1}^{*}$ associated with the signal $S^{*}$ :

$$
\frac{\epsilon}{2\left(x_{1}^{*}\right)^{2}}<Q<W_{11}^{E}\left(\frac{W_{11}^{E}}{S^{*}+\epsilon+1}-3\right)
$$

with $\epsilon=\tau_{1} / \tau_{2}$. Slow enough negative feedback $\left(\epsilon<\epsilon_{c}\right)$ is then necessary to destabilize one stable fixed point through a Hopf bifurcation. In particular, two stable fixed points can be 
destabilized simultaneously, one by a Hopf bifurcation and the other by a saddle-node bifurcation.

A last case study is to consider a nonlinear activation of the negative regulator as a step function $\mathrm{H}$ whose threshold $x_{0}$ lies between the two branches of steady state solutions of the uncoupled first component. The two saddle-node bifurcations arise for the values of $S$ and $x_{1}$ that satisify $F\left(S, x_{1}, x_{2}\right)=0$ and $\mathrm{d} F / \mathrm{d} x_{1}\left(S, x_{1}, x_{2}\right)=0$ with $x_{2}=0$ or $c_{0}$ respectively. As a result, the two saddle-node bifurcations occur at the same value of $S$ for two different values of $x_{1}, x_{1}^{+}$and $x_{1}^{-}\left(x_{1}^{+}>x_{1}^{-}\right)$, which satisfies the equality $\left(x_{1}^{+}-x_{1}^{-}\right)\left(2-x_{1}^{+}-x_{1}^{-}\right)=Q / W_{11}^{E}$. Such a coincidence of two saddle-node bifurcations corresponds to a transition from type-II bistability to oscillations.

\section{Appendix D. Measure of switching speed}

To define a measure of switching speed, we assume that the dynamical system (equation (1) rewritten as $\mathrm{d} \mathbf{x} / \mathrm{d} t=\mathbf{F}(\mathbf{x})$ ) is at a saddle-node bifurcation point $\mathbf{x}^{\mathrm{sn}}$ where a small perturbation can switch the system to another stable state $\mathbf{x}^{\text {st }}$, and we focus on the switching behavior of the master component $x_{1}$. The speed during the transition from low- to high- $x_{1}$ activities is correlated with phase-space velocity along the heteroclinic orbit connecting the saddle-node fixed point to the stable fixed point. We therefore define the switching time as the time required to cover some significant portion of the heteroclinic orbit $\gamma$ connecting the saddle-node and the stable fixed point:

$$
T_{\epsilon}=\int_{\gamma_{\epsilon}} \mathbf{d x} / \mathbf{F}(\mathbf{x})
$$

where $\gamma_{\epsilon}$ writes (for $x_{1}^{\mathrm{sn}}<x_{1}^{\mathrm{st}}$ )

$$
\gamma_{\epsilon}=\gamma \cap\left\{x_{1}<x_{1}^{\mathrm{st}}-\epsilon\right\} \cap\left\{x_{1}>x_{1}^{\mathrm{sn}}+\epsilon\right\} .
$$

The switching speed can be derived by dividing the switching distance by the switching time:

$$
v_{\epsilon}=\frac{\left|x_{1}^{\mathrm{st}}-x_{1}^{\mathrm{sn}}\right|-2 \epsilon}{T_{\epsilon}} .
$$

\section{References}

[1] Novick A and Weiner M 1957 Enzyme induction as an all-or-none phenomenon Proc. Natl Acad. Sci. USA $43553-66$

[2] Ferrell J E Jr and Machleder E M 1998 The biochemical basis of an all-or-none cell fate switch in Xenopus oocytes Science 280 895-8

[3] Murray A W and Kirschner M W 1989 Dominoes and clocks: the union of two views of the cell cycle Science 246 614-21

[4] Laurent M and Kellershohn N 1999 Multistability: a major means of differentiation and evolution in biological systems Trends Biochem. Sci. 24 418-22

[5] Ozbudak E M, Thattai M, Lim H N, Shraiman B I and Van Oudenaarden A 2004 Multistability in the lactose utilization network of Escherichia coli Nature 427 737-40

[6] Huang S, Eichler G, Bar-Yam Y and Ingber D E 2005 Cell fates as high-dimensional attractor states of a complex gene regulatory network Phys. Rev. Lett. 94128701
[7] Thomas R 1981 On the relation between the logical structure of systems and their ability to generate multiple steady states or sustained oscillation Springer Ser. Synergetics 9 $180-93$

[8] Plahte E, Mestl T and Onholt W S 1995 Feedback circuits, stability and multistationarity in dynamics systems J. Biol. Syst. 3 409-13

[9] Ferrell J E Jr 2002 Self-perpetuating states in signal transduction: positive feedback, double-negative feedback and bistability Curr. Opin. Cell Biol. 14 140-8

[10] Smits W K, Kuipers O P and Veening J W 2006 Phenotypic variation in bacteria: the role of feedback regulation Nat. Rev. Microbiol. 4 259-71

[11] Mitrophanov A Y and Groisman E A 2008 Positive feedback in cellular control systems Bioessays $30542-55$

[12] Kussell E and Leibler S 2005 Phenotypic diversity, population growth, and information in fluctuating environments Science 309 2075-8

[13] Kashiwagi A, Urabe I, Kaneko K and Yomo T 2006 Adaptive response of a gene network to environmental changes by fitness-induced attractor selection PLOS ONE 1 e 49

[14] Lopez-Maury L, Marguerat S and Bahler J 2008 Tuning gene expression to changing environments: from rapid responses to evolutionary adaptation Nat. Rev. Genet. 9 583-93

[15] Acar M, Becskei A and van Oudenaarden A 2005 Enhancement of cellular memory by reducing stochastic transitions Nature 435 228-32

[16] Süel G M, Kulkarni R P, Dworkin J, Garcia-Ojalvo J and Elowitz M B 2007 Tunability and noise dependence in differentiation dynamics Science 315 1716-9

[17] Kim D, Kwon Y K and Cho K H 2007 Coupled positive and negative feedback circuits form an essential building block of cellular signaling pathways Bioessays 29 85-90

[18] Grossman A D 1995 Genetic networks controlling the initiation of sporulation and the development of genetic competence in Bacillus subtilis Annu. Rev. Genet. 29 477-508

[19] Kaniak A, Xue Z, Macool D, Kim J H and Johnston M 2004 Regulatory network connecting two glucose signal transduction pathways in Saccharomyces cerevisiae Eukaryot. Cell 3 221-31

[20] Lai K, Robertson M J and Schaffer D V 2004 The sonic hedgehog signaling system as a bistable genetic switch Biophys. J. 86 2748-57

[21] Harris S L and Levine A J 2005 The p53 pathway: positive and negative feedback loops Oncogene 24 2899-908

[22] Bardwell L 2005 A walk-through of the yeast mating pheromone response pathway Peptides 26 339-50

[23] Zhang C C, Laurent S, Sakr S, Peng L and Bedu S 2006 Heterocyst differentiation and pattern formation in cyanobacteria: a chorus of signals Mol. Microbiol. 59 367-75

[24] Haberland M, Arnold M A, McAnally J, Phan D, Kim Y and Olson E N 2007 Regulation of HDAC9 gene expression by MEF2 establishes a negative-feedback loop in the transcriptional circuitry of muscle differentiation Mol. Cell Biol. 27 518-25

[25] Laub M T, Shapiro L and McAdams H H 2007 Systems biology of Caulobacter Annu. Rev. Genet. 41 429-41

[26] Rubinstein A, Gurevich V, Kasulin-Boneh Z, Pnueli L, Kassir Y and Pinter R Y 2007 Faithful modeling of transient expression and its application to elucidating negative feedback regulation Proc. Natl. Acad. Sci. USA $1046241-6$

[27] Benayoun B A, Batista F, Auer J, Dipietromaria A, L'Hote D, De Baere E and Veitia R A 2009 Positive and negative feedback regulates the transcription factor FOXL2 in response to cell stress: evidence for a regulatory imbalance 
induced by disease-causing mutations Hum. Mol. Genet. 18 632-44

[28] Boissonade J and De Kepper P 1980 Transitions from bistability to limit cycle oscillations. Theoretical analysis and experimental evidence in an open chemical system J. Phys. Chem. 84 501-6

[29] Epstein I R and Pojman J A 1998 An Introduction to Nonlinear Chemical Dynamics Oscillations, Waves, Patterns, and Chaos (Oxford: Oxford University Press)

[30] Gillespie D T 2000 The chemical langevin equation J. Chem. Phys. 113 297-306

[31] Voigt C A, Wolf D M and Arkin A P 2005 The Bacillus subtilis sin operon: an evolvable network motif Genetics 169 1187-202

[32] Song H, Smolen P, Av-Ron E, Baxter D A and Byrne J H 2007 Dynamics of a minimal model of interlocked positive and negative feedback loops of transcriptional regulation by cAMP-response element binding proteins Biophys. $J$. 92 3407-24

[33] Agrawal S, Archer C and Schaffer D V 2009 Computational models of the Notch network elucidate mechanisms of context-dependent signaling PLoS Comput. Biol. 5 e 1000390

[34] Guantes R and Poyatos J F 2006 Dynamical principles of two-component genetic oscillators PLoS Comput. Biol. 2 e 30

[35] Tsai T Y, Choi Y S, Ma W, Pomerening J R, Tang C and Ferrell J E Jr 2008 Robust, tunable biological oscillations from interlinked positive and negative feedback loops Science 321 126-9

[36] Krishna S, Semsey S and Jensen M H 2009 Frustrated bistability as a means to engineer oscillations in biological systems Phys. Biol. 636009

[37] Krishna S, Semsey S and Sneppen K 2007 Combinatorics of feedback in cellular uptake and metabolism of small molecules Proc. Natl. Acad. Sci. USA $10420815-9$

[38] Meinhardt $\mathrm{H}$ and Gierer A 2000 Pattern formation by local self-activation and lateral inhibition Bioessays 22 753-60
[39] Brandman O, Ferrell J E Jr, Li R and Meyer T 2005 Interlinked fast and slow positive feedback loops drive reliable cell decisions Science 310 496-8

[40] Huang S, Guo Y P, May G and Enver T 2007 Bifurcation dynamics in lineage-commitment in bipotent progenitor cells Dev. Biol. 305 695-13

[41] Pfeuty B, David-Pfeuty T and Kaneko K 2008 Underlying principles of cell fate determination during $\mathrm{G} 1$ phase of the mammalian cell cycle Cell Cycle 7 3246-57

[42] Guantes R and Poyatos J F 2008 Multistable decision switches for flexible control of epigenetic differentiation PLoS Comput. Biol. 4 e1000235

[43] Rosenfeld N, Elowitz M B and Alon U 2002 Negative autoregulation speeds the response times of transcription networks J. Mol. Biol. 323 785-93

[44] Ozcan S and Johnston M 2000 Function and regulation of yeast hexose transporters Microbiol. Mol. Biol. Rev. 63 554-69

[45] Losick R and Desplan C 2008 Stochasticity and cell fate Science 320 65-8

[46] Acar M, Mettetal J T and van Oudenaarden A 2008 Stochastic switching as a survival strategy in fluctuating environments Nature Genetics 40 471-5

[47] Maughan H and Nicholson W L 2004 Stochastic processes influence stationary-phase decisions in Bacillus subtilis J. Bacteriol. $1862212-4$

[48] Veening J W, Smits W K, Hamoen L W and Kuipers O P 2006 Single cell analysis of gene expression patterns of competence development and initiation of sporulation in Bacillus subtilis grown on chemically defined media J. Appl. Microbiol. 101 531-41

[49] Morgan D O 2007 The Cell Cycle: Principles of Control (London: New Science Press)

[50] Gonze D and Goldbeter A 2001 A model for a network of phosphorylation-dephosphorylation cycles displaying the dynamics of dominoes and clocks J. Theor. Biol. $210167-86$

[51] Tyson J J and Novák B 2008 Temporal organization of the cell cycle Curr. Biol. 18 759-68

[52] Alon U 2006 An Introduction to Systems Biology: Design Principles of Biological Circuits (Boca Raton, FL: CRC Press) 